\title{
A new blast wave facility at IUSTI Marseille
}

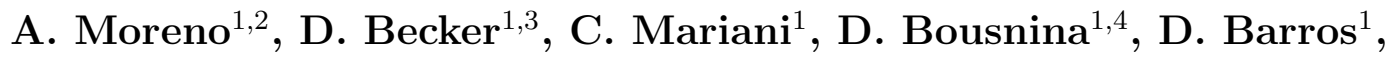 \\ A. Marty ${ }^{1}$, J. Massoni ${ }^{1}$, D. Leriche ${ }^{5}$, G. Jourdan ${ }^{1}$, L. Houas ${ }^{1}$ \\ ${ }^{1}$ Aix-Marseille Univ, CNRS, IUSTI, Marseille, France \\ ${ }^{2}$ ENSTA Bretagne, 29200 Brest, France \\ ${ }^{3}$ INP Bordeaux Aquitaine, 33600 Pessac, France \\ ${ }^{4}$ Ecole Polytechnique de Tunisie, Carthage, Tunisie \\ ${ }^{5}$ DGA Techniques navales, Avenue de la Tour Royale, 83050 Toulon, France \\ Corresponding Author's name: lazhar.houas@univ-amu.fr
}

\begin{abstract}
A long duration blast wave facility was built in order to study human and material damages at distances relatively far from the epicenter of a high explosion. The blast tube allows one to generate weak blast waves with a maximum pressure ranging from 5 to 1000 mbar, and long time duration of the positive phase up to few hundreds of milliseconds. After about 150 runs, results show that the time duration of the propagation of the rarefaction waves in the blast tube is strongly connected to the time duration of the positive phase of the blast wave. We also found that the duration of the positive phase is weakly affected as one moves away from the tube exit.
\end{abstract}

\section{Introduction}

To study long-duration blast waves in a controlled laboratory environment, the IUSTI Laboratory developed a new blast wave facility.

The ability to protect people, structures and properties from explosions is a major global challenge. The blast wave issued from an explosion is at the center of these concerns, given its potential to cause damage at distances relatively far from the epicenter of the explosion [1]. This impact, however, is not completely understood or controlled, and is therefore the subject of many research studies.

Such a wave is indeed in the category of long-duration blast waves, whose duration of the positive phase lasts more than $60 \mathrm{~ms}$ and may be representative of a blast wave resulting from an explosion taking place in a nuclear power plant few dozen kilometers away.

Several facilities have been built in the past [2], [3], [4], [5] with various blast wave characteristics. The Sandia National Laboratory blast tube [2] generates blast waves with $P_{\text {Max }}=4.1$ bar (inside the tube), a total length of about $L=37 \mathrm{~m}$ (variable) and a time duration of the positive phase (from start to return to the atmospheric pressure) of $\Delta t=$ $40 \mathrm{~ms}$. The College of Wisconsin [3] uses helium as high pressure gas for $P_{\text {Max }}=4.5$ bar, $L=3.3 \mathrm{~m}$ and $\Delta t=0.18 \mathrm{~ms}$. The Norwegian University of Science and Technology blast tube characteristics are $P_{M a x}=3.2 \mathrm{bar}, L=16.97 \mathrm{~m}$ and $\Delta t=30 \mathrm{~ms}$. And the blast tube at the University of Mississippi reaches about $P_{\text {Max }}=1000$ bar, for $L=6.3 \mathrm{~m}$ and $\Delta t=50 \mathrm{~ms}$.

As reported in the following paper, the IUSTI blast tube was built to obtain weaker and 

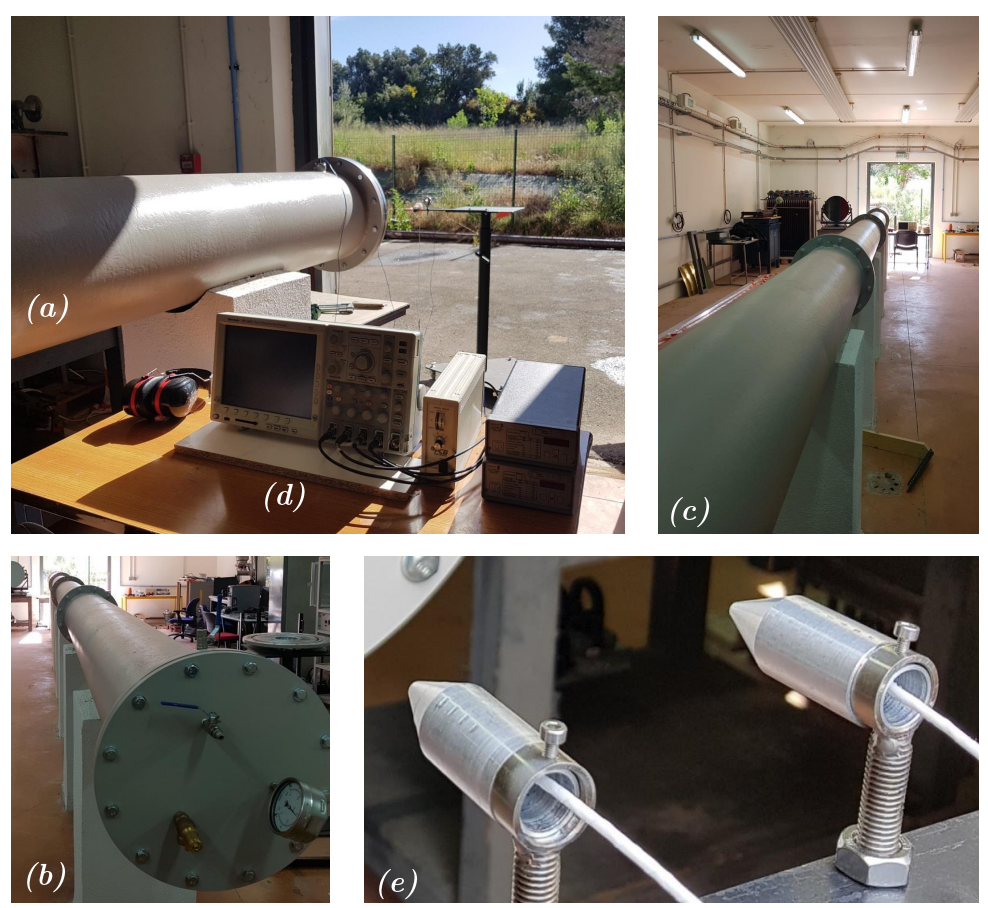

Fig. 1 Views of the new blast tube at IUSTI Marseille: (a), (b), (c) blast tube, (d) Tektronix DPO4054 oscilloscope and pressure gauge transducers, and (e) Kulite XTL190M-13.5BARA external pressure gauges.

lasting longer blast waves with 5 mbar $\leq P_{M a x} \leq 1000$ mbar, for $3 \mathrm{~m} \leq L \leq 12 \mathrm{~m}$ and $10 \mathrm{~ms} \leq \Delta t \leq 500 \mathrm{~ms}$.

\section{T355 blast wave facility and experimental setup}

The IUSTI Marseille blast tube consists of 4 sections of $3 \mathrm{~m}$ each with a diameter of $355 \mathrm{~mm}$. All the experiments presented in this work were conducted with a total length of $12 \mathrm{~m}$, as shown in Fig. 1. It has different exit membrane diameters ranging from 2 to $30 \mathrm{~cm}$ (Fig. 2). Currently, the pressure discharge ranges from 0.1 to 1 bar, but this value can be tuned larger. At the tube exit, 1, 2, 3 or 4 aluminum sheets are used as diaphragm for generating the blast waves. This makes possible the generation of both weak and long time duration blast waves. T355 blast tube is equipped with two 113B26 PCB piezoelectric pressure gauges located inside the tube at $x-9.5 \mathrm{~cm}$ and $x-290.5 \mathrm{~cm}$ ( $x=0$ corresponds to the location of the diaphragm) and two XTL-190M Kulite piezoresistive sensors located outside the tube and variable from $x=25 \mathrm{~cm}$ to $x=150 \mathrm{~cm}$. A Tektronix DPO4054 oscilloscope allows to record the pressure signals.

A wave diagram and a typical pressure-time blast wave profile, showing peak overpressure (which is relative to the atmospheric pressure) and positive phase duration is shown in Figs. 3 and 4. 

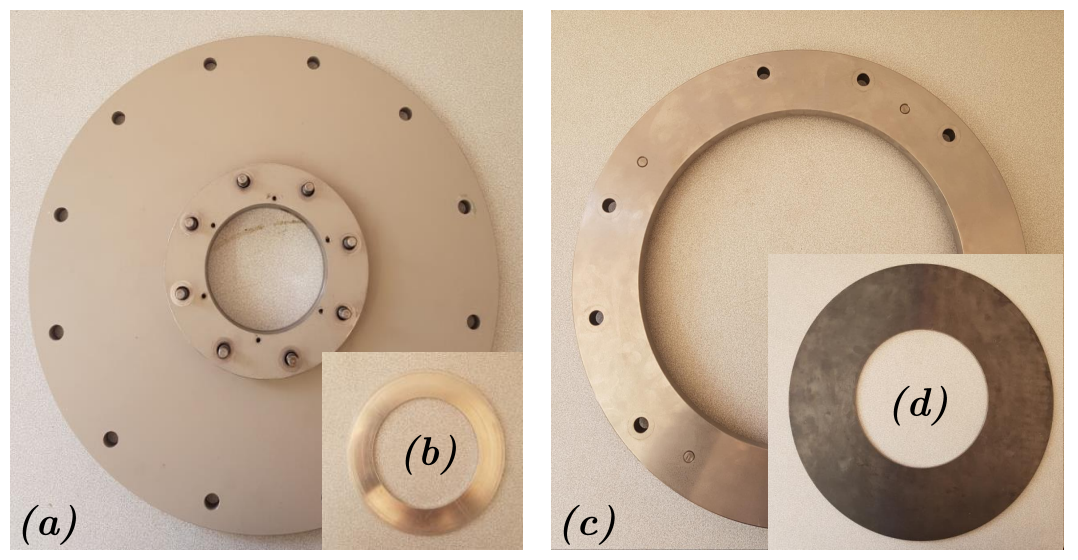

Fig. 2 (a-b) Small membrane supports $(2 \mathrm{~cm}, 4 \mathrm{~cm}, 6 \mathrm{~cm}, 8 \mathrm{~cm}$ and $10 \mathrm{~cm}$ in diameter), and (c-d) large membrane supports $(20 \mathrm{~cm}, 25 \mathrm{~cm}$ and $30 \mathrm{~cm}$ in diameter).

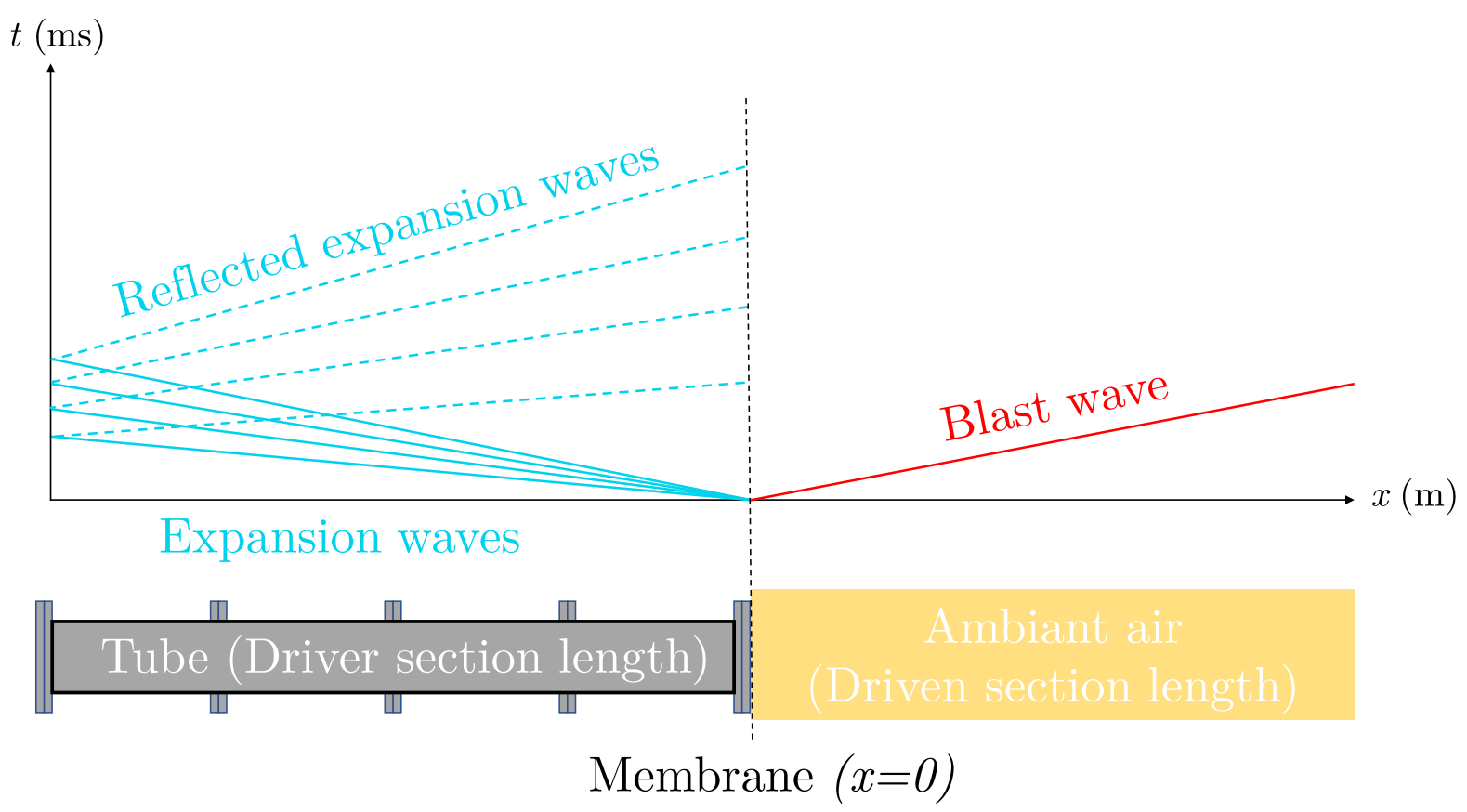

Fig. 3 Wave diagram. 


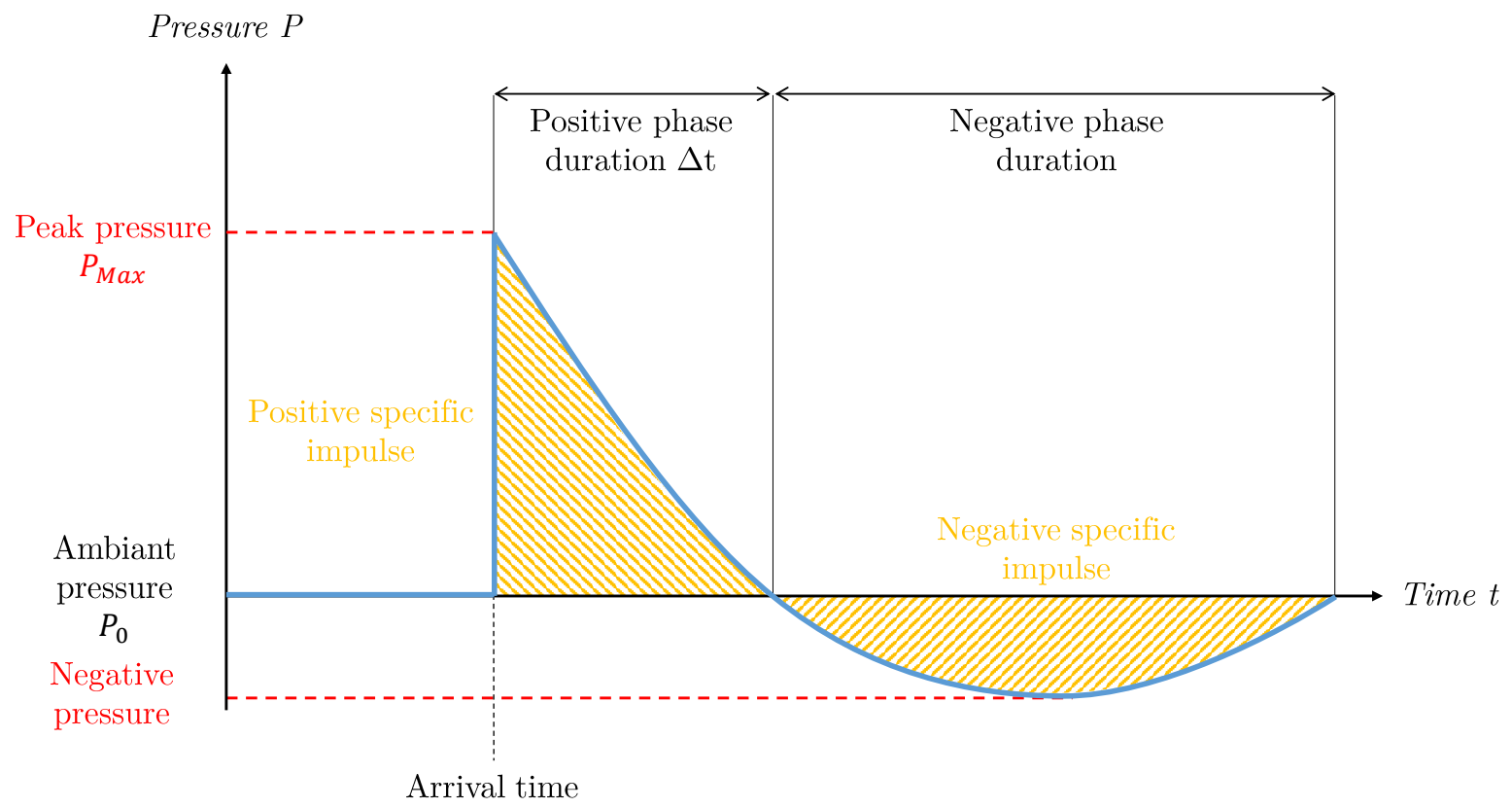

Fig. 4 Theoretical ideal signal of a blast wave.

\section{Results}

A total of 150 runs were performed for various initial conditions i.e. the membrane rupture pressure $P_{\text {Rupt }}$, the membrane diameter $\phi_{\text {Membrane }}$, and the number of aluminum foils. For each run, we recorded four signals, the pressure within the blast tube (with two PCB transducers) and the total pressure outside the tube (with two Kulite transducers) within the blast tube axis $(r=0 \mathrm{~cm})$ and shifted from the axis of the tube $(4 \mathrm{~cm} \leq r \leq$ $20 \mathrm{~cm})$.

Three runs, conducted for the same initial conditions at different distances from the blast tube exit, are discussed here. Fig. 4 presents the results obtained for runs T355\#122, T355\#124 and T355\#131. The blue signal represents the evolution of the pressure inside the blast tube $9.5 \mathrm{~cm}$ upstream of its exit section. We observe about three round-trip of the rarefaction waves before the pressure starts to increase for reaching the atmospheric pressure. The red signal gives the response of the blast wave centered on the blast tube axis for different distances, $100 \mathrm{~cm}, 125 \mathrm{~cm}$ and $138 \mathrm{~cm}$, from the membrane (the exit of the tube) position. The yellow signal records the same phenomenon but shifted from $8 \mathrm{~cm}$ or $18 \mathrm{~cm}$ from the blast tube axis. This allows us to estimate the decrease in pressure as we move away from the axis of the tube for the considered initial conditions. We observe that the blast wave generated pressure is about $P_{\text {Max }}=100$ mbar along the blast tube axis and decreases to about 50 mbar $8 \mathrm{~cm}$ further.

The same experiment, at a distance of $125 \mathrm{~cm}$ (Figs. 4c and 4d), gives a pressure of about $P_{\text {Max }}=50$ mbar for $r=0 \mathrm{~cm}$ and 30 mbar for $r=8 \mathrm{~cm}$. Finally, continuing to move away from the exit of the blast tube at a distance of $138 \mathrm{~cm}$, (see Figs. 4e and $4 \mathrm{f}$ ), the pressure is quite the same on the blast tube axis, $P_{M a x}=50$ mbar for $r=0 \mathrm{~cm}$, but strongly decreases to less than 10 mbar when we shift of $18 \mathrm{~cm}$ from the blast tube axis. 


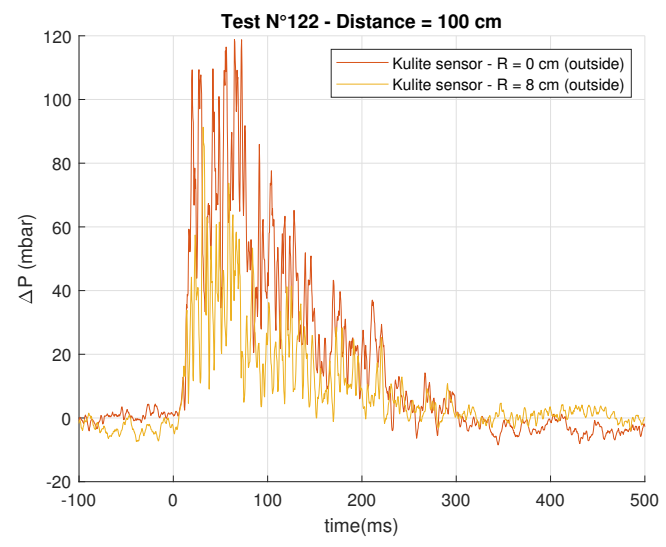

(a)

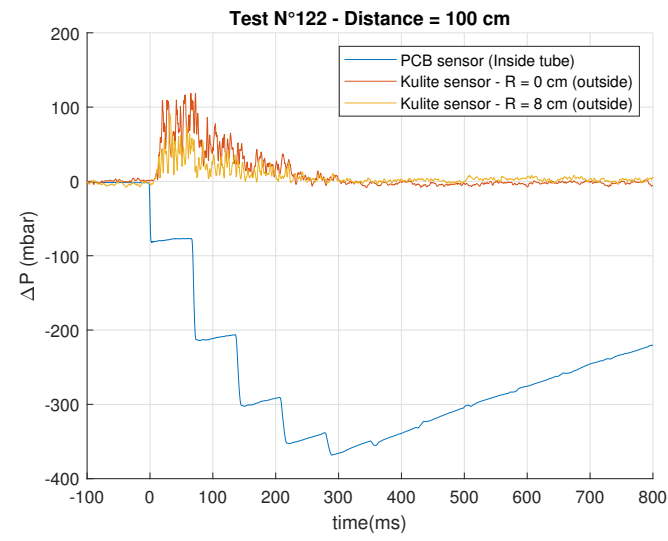

(b)

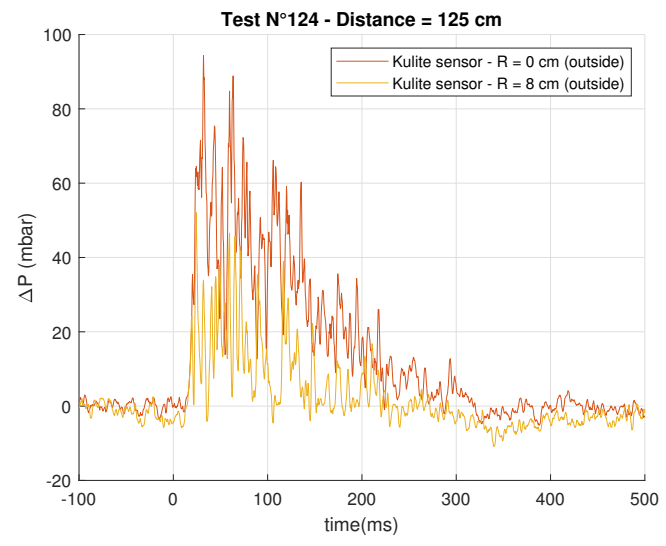

(c)

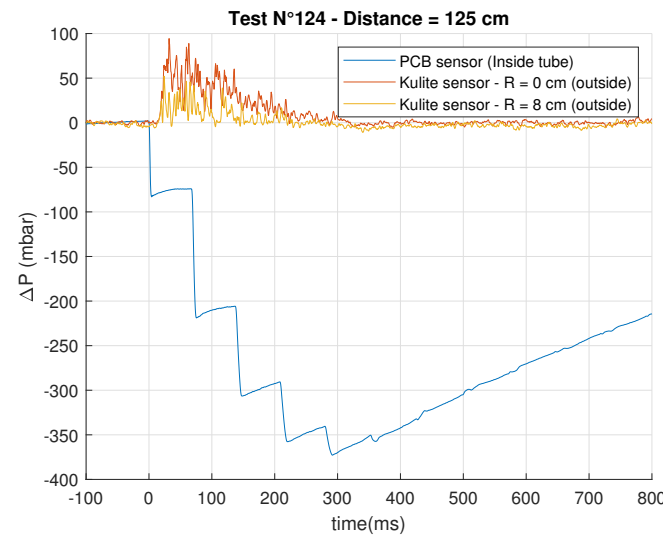

(d)

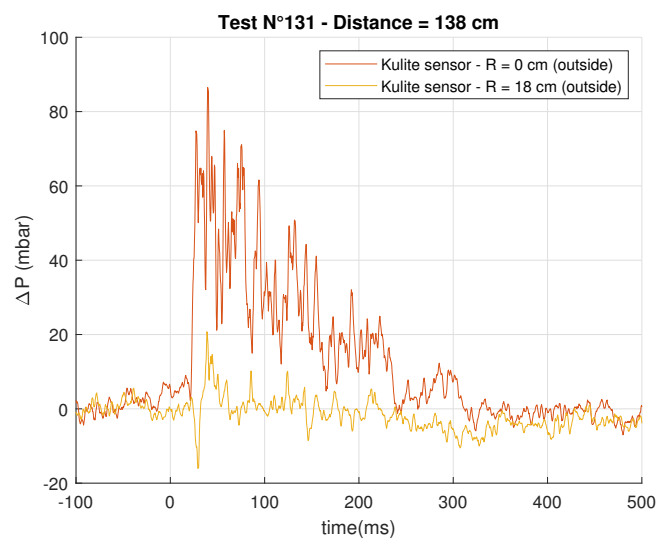

(e)

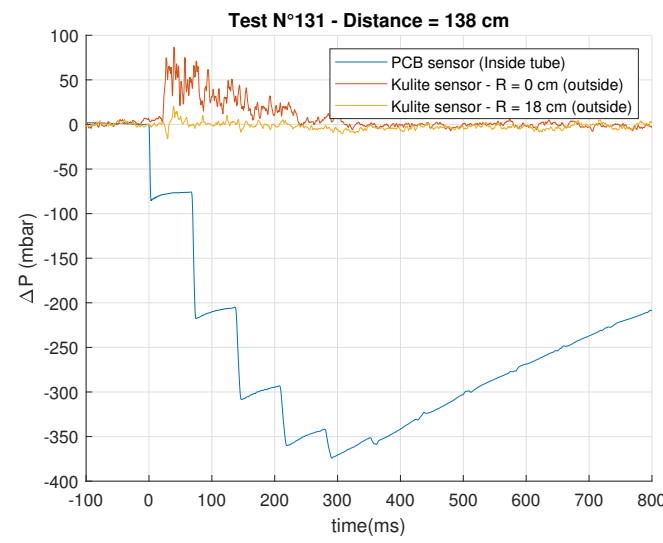

(f)

Fig. 5 Results obtained with $P_{\text {Rupt }}=1.4$ bar, $\Delta t=300 \mathrm{~ms}, \phi_{\text {Membrane }}=10 \mathrm{~cm}, 3$ aluminum foils for a distance of $100 \mathrm{~cm}$ from the exit of the tube (a,b), $125 \mathrm{~cm}(\mathrm{c}, \mathrm{d})$ and $138 \mathrm{~cm}(\mathrm{e}, \mathrm{f})$.

In addition, we point out that, for the majority of the experiments, the time duration of the propagation of the rarefaction waves in the blast tube is very close to the time 
duration of the positive phase of the blast wave: in the present experiments is close to $\Delta t=300 \mathrm{~ms}$.

Finally, it seems that the duration of the positive phase is weakly influenced by the distance to the exit of the tube, and rather seems to be related to the combined effects of the diameter and the resistance of the membrane, which is linked to the rupture pressure $P_{\text {Rupt }}$.

\section{Conclusion}

A new blast tube was designed in order to obtain weak blast waves characterized by both a peak pressure ranging from tens to hundreds of millibars and a duration of the positive phase up to hundreds milliseconds. First results show that the time duration of the propagation of the rarefaction waves in the blast tube is very close to the time duration of the positive phase of the blast wave. Furthermore, it appears that the duration of the positive phase is only slightly affected by the distance to the tube exit.

\section{Acknowledgements}

This work is supported by DGA/TN under contract \#2018830026.

\section{References}

[1] D. R. Kirk, J. M. Faure and H. Gutierrez, Generation and analysis of blast wave from compressed air-driven shock tube, Proceedings of the 44thAIAA/ASME/SAE/ASEE Joint Propulsion Conference Exhibit, In 21-23 July, Hartford CT, 2008.

[2] Sandia National Laboratory blast tube (private communication).

[3] M. D. Budde, A. Shah, M. McCrea, W. E. Cullinan, F. A. Pintar and B. D. Stemper, Primary blast traumatic brain injury in the rat: relating diffusion tensor imaging and behavior, Frontiers in NEUROLOGY, Vol. 4, 00154, 2013.

[4] V. Aune, E. Fagerholt, M. Langseth and T. Borvik, A shock tube facility to generate blast loading on structures, International, vol. 7(3) 340-366, 2016.

[5] Blast Tube at the University of Mississippi (private communication). 